\title{
ON THE UNIQUENESS OF THE PROLONGATION OF AN OPEN RIEMANN SURFACE OF FINITE GENUS ${ }^{1}$
}

\author{
KÔTARO OIKAWA
}

1. Let $F$ be an open Riemann surface of finite genus $g$. Suppose there exists a Riemann surface $W$ with the property that there exists a conformal mapping $f$ of $F$ onto a proper subregion of $W$. Then $W$ or, more precisely, the pair $(W, f)$ is called a prolongation of $F$.

In the following, we assume exclusively that $W$ is a closed surface of genus $g$. The existence of such a $(W, f)$ is well known [4]. When is such a prolongation determined uniquely? This is the problem that we shall discuss in the present paper.

Such a problem has been proposed by Nevanlinna [6]. In his case the uniqueness means the following: $F$ is said to admit a unique prolongation if, for any $\left(W_{1}, f_{1}\right)$ and $\left(W_{2}, f_{2}\right)$, the mapping $f_{2} \circ f_{1}^{-1}$ can be extended to a conformal mapping of $W_{1}$ onto $W_{2}$.

The Uniqueness Theorem. $F$ admits the unique prolongation in the above sense if and only if $F$ is of $O_{A D} .^{2}$

It was conjectured by Nevanlinna [6] and was proved by Ahlfors and Beurling [2] for $g=0$ and by Mori [5] for $g \geqq 1$.

2. We ask here the "uniqueness" in another sense: When are all the $W$ conformally equivalent to each other? Can the only-if-part of the Uniqueness Theorem be replaced by the following weaker form: If all the $W$ are conformally equivalent to each other, then $F$ is of $O_{A D}$ ? It is trivially not true for $g=0$; in general, even if $f_{2} \circ f_{1}^{-1}$ is not extendable to a conformal mapping of $W_{1}$ onto $W_{2}$, still they may be conformally equivalent. Nevertheless we have

THEOREM 1. Let $F$ be an open Riemann surface of finite genus $g \geqq 1$. Then all the closed Riemann surfaces of genus $g$ which are prolongations of $F$ are conformally equivalent to each other if and only if $F$ is of $O_{A D}$.

Its if-part is a direct consequence of the Uniqueness Theorem. To prove the only-if-part, we shall apply the theory of Teichmüller

Received by the editors November 16, 1959.

1 This paper was prepared under the Contract No. DA-30-069-1253, OOR Project No. 247, between the Institute of Mathematical Sciences, New York University and the Office of Ordnance Research, U. S. Army.

${ }^{2} O_{A D}$ is the class of Riemann surfaces which do not carry a nonconstant analytic function with finite Dirichlet integral. 
space $[8 ; 1 ; 3]$. For simplicity, we shall treat merely the case $g \geqq 2$; with a slight modification the reasoning is applicable also to the case $g=1$.

Let $T_{\theta}$ be the Teichmüller space of closed Riemann surfaces of genus $g$ (for the definition the reader is referred to, e.g., Ahlfors $\left[1\right.$, p. 53]). In our previous paper [7], we considered the set $P(F) \subset T_{0}$ which corresponds to the set of all the prolongations $(W, f)$ of $F$. Before proving Theorem 1, we shall prove

Theorem 2. Let $g \geqq 2$. If $F \in O_{A D}$ then $P(F)$ consists of a single point. If $F \in O_{A D}$ then $P(F)$ contains an interior point.

3. Proof of Theorem 2. The first part is a direct consequence of the Uniqueness Theorem.

To prove the second part suppose $F \notin O_{A D}$. Then it is not difficult to show that there exists a $(W, f)$ such that the set $E=W-f(F)$ has positive measure (see Mori [4] and Ahlfors-Beurling [2]). We can find a complex basis $\mu_{j}(d \bar{z} / d z)(j=1,2, \cdots, 3 g-3)$ of Beltrami differentials modulo trivial Beltrami differentials on $W$ (for the definition see Bers [3]) such that $\mu_{j} \equiv 0$ on $W-E$. In fact, let $\phi_{j} d z^{2}(j=1,2, \cdots, 3 g-3)$ be a complex basis of regular analytic quadratic differentials on $W$ such that

$$
\iint_{E} \frac{\phi_{j} \bar{\phi}_{k}}{L} d x d y=\delta_{j k}
$$

where $L|d z|^{2}$ is the Poincare metric on $W$; then

$$
\mu_{j}=\left\{\begin{array}{cl}
\bar{\phi}_{j} / L & \text { on } E \\
0 & \text { on } W-E
\end{array}\right.
$$

is the desired, because the local triviality of $\sum_{j=1}^{30-3} c_{j} \mu_{j}$ implies that $c_{j} \iint_{E}\left|\phi_{j}\right| d x d y=0$ and, therefore, $c_{j}=0(j=1,2, \cdots, 3 g-3)$ (this method is merely a modification of the one in Bers [3]). Let $W^{\mu}$ be the Riemann surface whose conformal structure is defined by the metric $d s=|d z+\mu d \bar{z}|$ on $W$. As is shown by Bers [3], the set $U$ $=\left\{W^{\mu} ; \mu=\sum_{j-1}^{30-3} c_{j} \mu_{j}, \sum_{j-1}^{30-3}\left|c_{j}\right|<\epsilon\right\}$ is an open set in $T_{q}$ if $\epsilon>0$ is sufficiently small. The conformal structure of $f(F)$ in any surface in $U$ is not changed. Hence every $\left(W^{\mu}, f\right), W^{\mu} \in U$, is a prolongation of $F$ and, therefore, $U \subset P(F)$.

4. Proof of Theorem 1. The Teichmüller space $T_{0}$ admits the projection $\pi$ in the well-known manner onto the space of conformal equivalence classes of closed Riemann surfaces of genus $g$. 
Suppose that all the prolongations $W$ of $F$ are conformally equivalent to each other. Then $\pi P(F)$ consists of a single point. It is fairly easy to see that, in this case, $P(F)$ is a discrete set. ${ }^{3}$ On the other hand we showed in [7] that $P(F)$ is a connected set. Therefore we see that $P(F)$ consists of a single point and, by Theorem 2, we conclude that $F$ is of $O_{A D}$.

\section{REFERENCES}

1. L. V. Ahlfors, On quasiconformal mappings, J. Analyse Math. vol. 3 (19531954) pp. 1-58, 207-208.

2. L. V. Ahlfors and A. Beurling, Conformal invariants and function-theoretic null-sets, Acta Math. vol. 83 (1950) pp. 101-129.

3. L. Bers, Spaces of Riemann surfaces, Proceedings of the Mathematical Congress, Edinburgh, 1958, pp. 349-369.

4. S. Bochner, Fortsetzung Riemannscher Flächen, Math. Ann. vol. 98 (1928) pp. 406-421.

5. A. Mori, A remark on the prolongation of Riemann surfaces of finite genus, J. Math. Soc. Japan vol. 4 (1952) pp. 198-209.

6. R. Nevanlinna, Eindeutigkeitsfragen in der Theorie der konformen Abbildung, Comptes Rendus 10, Congr. Scand., 1946.

7. K. Oikawa, On the prolongation of an open Riemann surface of finite genus, Kódai Math. Sem. Rep. vol. 9 (1957) pp. 34-41.

8. O. Teichmüller, Extremale quasikonforme Abbildungen und quadratische Differentiale, Abh. Preuss. Akad. Math.-Naturw. Kl. vol. 22 (1940) pp. 1-197.

Institute of Mathematical Sciences, New York University and ToKyo INSTITUTE OF TECHNOLOGY

${ }^{3}$ It is stated without proof in $[8$, p. 168]. It can be proved easily if the reasoning in $[3]$ is used. 